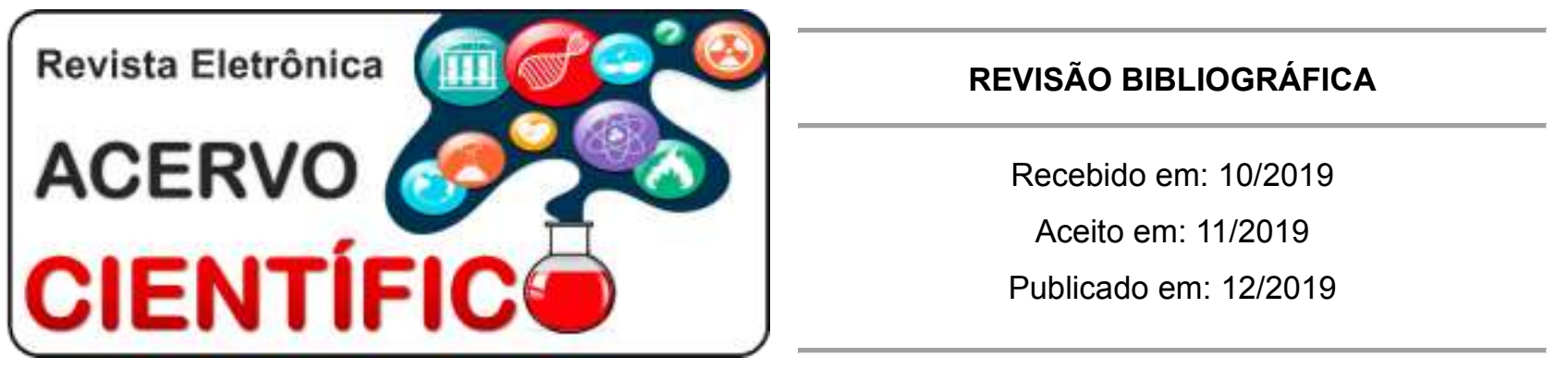

\title{
Frequência da resistência ao etambutol por Mycobacterium Tuberculosis: Uma revisão de literatura
}

Frequency of resistance to ethambutol by Mycobacterium Tuberculosis: A literature review

Frecuencia de resistencia al etambutol por Mycobacterium Tuberculosis: Una revisíon de literatura

Romário Torres Alcântara ${ }^{1 *}$, Crysvânia Santos Nascimento', Camila Conceição Santos Temóteo, João Paulo Siqueira Correia ${ }^{1}$, Alice Barbosa Nascimento ${ }^{1}$, Lúcia Gabriela Costa Silva ${ }^{1}$, José Aderval Aragão'.

Resumo: O objetivo deste estudo foi realizar uma revisão de literatura acerca da frequência da resistência ao etambutol em casos ou amostras por Mycobacterium Tuberculosis. Um protocolo de pesquisa foi formalizado e executado para conduzir a pesquisa no banco de dados do PubMed durante julho de 2019 e setembro de 2019, com artigos publicados entre o ano de 2014 e 2019. Os resultados obtidos foram analisados e incorporados ao presente estudo a partir de critérios de inclusão e exclusão estabelecidos no protocolo. Assim, se buscou elucidar acerca da frequência da resistência tuberculínica ao etambutol e como resultado se constatou uma baixa frequência de resistência ao medicamento em questão em relação aos demais medicamentos estabelecidos como de primeira linha, de acordo com o local do estudo, para o tratamento da Tuberculose.

Palavras-chave: Resistência microbiana a medicamentos, Etambutol, Tuberculose.

Abstract: The aim of this study was to perform a literature review about the frequency of ethambutol resistance in cases or samples by Mycobacterium Tuberculosis. A research protocol was formalized and executed to conduct the search in the PubMed database during July 2019 and September 2019, with articles published between 2014 and 2019. The results obtained were analyzed and incorporated into the present study from inclusion and exclusion criteria set out in the protocol. Thus, we sought to elucidate about the frequency of tuberculin resistance to ethambutol and as a result we found a low frequency of resistance to the drug in question in relation to the other drugs established as first line, according to the study site, for the treatment of Tuberculosis.

Keywords: Drug resistance, Ethambutol, Tuberculosis.

Resumen: El objetivo de este estudio fue realizar una revisión de la literatura sobre la frecuencia de resistencia al etambutol en casos o muestras de Mycobacterium Tuberculosis. Se formalizó y ejecutó un protocolo de investigación para realizar la búsqueda en la base de datos PubMed durante julio de 2019 y

${ }^{1}$ Universidade Tiradentes (UNIT), Aracaju-Sergipe. *E-mail: romalcmed@gmail.com 
septiembre de 2019, con artículos publicados entre 2014 y 2019. Los resultados obtenidos se analizaron e incorporaron al presente estudio a partir de Criterios de inclusión y exclusión establecidos en el protocolo. Por lo tanto, buscamos dilucidar sobre la frecuencia de resistencia de la tuberculina al etambutol y, como resultado, encontramos una baja frecuencia de resistencia al medicamento en cuestión en relación con los otros medicamentos establecidos como primera línea, según el sitio del estudio, para el tratamiento de Tuberculosis.

Palabras clave: Farmacorresistencia microbiana, Etambutol, Tuberculosi.

\section{INTRODUÇÃO}

A tuberculose (TB) trata-se de uma doença causada pela Mycobacterium tuberculosis também denominado de Bacilo de Kock (BK). A transmissão ocorre através de partículas de aerossóis contendo o patógeno, o que facilita a disseminação da doença. O longo período de latência entre a infecção inicial e a apresentação clínica, é uma das características marcantes. É importante destacar um acometimento preferencial dos pulmões, o que caracteriza a forma pulmonar da doença. Ademais, pode manifestar-se em outros órgãos do corpo como ossos, rins e meninges, representando sua forma extrapulmonar, caracterizada por uma resposta granulomatosa associada à intensa inflamação e lesão tissular (NOGUEIRA AF, et al., 2012).

No período de 2001 e 2014, no Brasil, foi notificado e confirmado mais de um milhão de novos casos de Tuberculose; desses, cerca de setenta mil culminaram em óbitos (SILVA MEND, et al., 2018). Em geral, um número relativamente pequeno de pessoas infectadas com $\mathrm{M}$. tuberculosis desenvolve a doença, no entanto, a probabilidade de desenvolver a tuberculose é muito maior entre as pessoas infectadas pelo HIV. Tem acometimento preferencial no sexo masculino e afeta principalmente adultos nas faixas etárias economicamente produtivas (WHO, 2013). Para Li Y et al. (2016), a ocorrência generalizada de TB resistente a medicamentos (DR) (DR-TB), especialmente a TB multirresistente (MDR) (MDR-TB), tornou-se uma importante preocupação de saúde pública global.

No aspecto clínico, a maioria dos indivíduos, tem uma infecção de curso assintomática, a qual manifestase com febre e dor torácica do tipo pleurítica. No adulto o quadro apresenta, inicialmente, sintomas inespe-cíficos, como febre diurna, sudorese noturna, perda ponderal, anorexia, mal-estar e fraqueza. Com a evolução do quadro, os pacientes desenvolvem tosse produtiva, eventualmente com hemoptise maciça. Um exame de imagem auxiliar no diagnóstico é a radiografia de tórax, a qual deve ser solicitada em todo caso suspeito, porém, apresenta-se dentro dos padrões de normalidade em $15 \%$ dos casos. As alterações mais comuns no exame são: opacidades paren-quimatosas, linfonodomegalia, atelectasia, padrão miliar e derrame pleural. (SILVA MEND, et al., 2018; NOGUEIRA AF, et al., 2012).

O método mais comum para o diagnóstico TB no mundo é a baciloscopia de escarro, em que amostras de bactérias são observadas no escarro e examinadas ao microscópio. Após recentes avanços no diagnóstico da TB, atualmente, o uso de métodos moleculares para o diagnóstico e avaliação de TB resistente a medicamentos é mais utilizado, visto que consegue avaliar a sensibilidade e especificidade das cepas resistentes aos fármacos utilizados. Com isso, contribui para um tratamento efetivo, tendo em vista que será atingida a resposta terapêutica almejada (CHENG B, et., 2014).

Para Kim HJ, et al. (2017), é importante conhecer os tratamentos efetivos para uma doença tão incidente e prevalente quanto a tuberculose, buscando-se assim uma menor taxa de resistência à terapêutica. Atualmente, são empregados para o tratamento agentes de primeira escolha, os quais foram encontrados eficácia favorável e nível de toxicidade aceitável, são estes a isoniazida, a rifampicina, o etambutol e a pirazinamida. Como segunda escolha para o tratamento, os fármacos utilizados em casos de resistência àqueles anteriormente citados, incluem a amicacina, a capreomicina, o ciprofloxacino, a cicloserina, a etionamida, a canamicina, o ofloxacino, o ácido paminosalicílico e a protionamida (SILVA MEND, et al., 2018; NOGUEIRA AF, et al., 2012). 
O etambutol é um dos medicamentos de primeira linha no tratamento da Tuberculose. (TAKAYAMA K et al. 1979; $X U$ Y et al. 2015). No entanto, existem diversas cepas resistentes à este fármaco. (COBAN AY et al., 2014; FENG Y et al., 2013; SAFI H et al.,2013).

De forma semelhante a taxa de resistência ao medicamento aumenta gradativamente em algumas regiões e se aproxima de $50 \%$ em pacientes que estão sendo submetidos novamente ao tratamento. (SANGARÉ L et al., 2010; WU MH et al., 2013).

Assim, tais fatos podem levar a uma interferência no diagnóstico e principalmente no tratamento, o que requer a amplificação dos estudos quanto às mutações genéticas relacionadas a resistência ao etambutol (CUI Z et al., 2014).

Desta forma, o objetivo foi revisar, na literatura, a cerca de identificar a frequência da resistência ao antibacteriano etambutol em casos ou amostras por Mycobacterium tuberculosis.

\section{MÉTODOS}

Para responder às perguntas especificadas, usamos as palavras-chaves selecionadas drug resistance, ethambutol, tuberculosis e o seguinte banco de dados: PubMed (US National Library of Medicine/National Institutes of Health). Foram considerados todos os estudos encontrados na área de pesquisa em questão que foram publicados no período de 2014 até 2019.

A revisão da literatura foi realizada de julho de 2019 a setembro de 2019. A busca para materiais que abordam a frequência da resistência ao etambutol em pacientes com tuberculose resultou 58 artigos. Do total de artigos coletados, após implementados os critérios de inclusão e exclusão, 15 artigos foram selecionados.

No protocolo estabelecido para a etapa de planejamento, foram especificadas as seguintes questões de pesquisa: "Qual a frequência da resistência ao antibacteriano etambutol em casos ou amostras por Mycobacterium tuberculosis?" e "Qual é a frequência de resistência ao Etambutol relacionado a outros microbianos quando presentes?"

A revisão foi realizada em três etapas: (i) planejamento, em que as diretrizes de pesquisa foram baseadas em um protocolo; (ii) condução, que consistia em executar a busca e seleção de obras de interesse de acordo com a inclusão e exclusão e critérios definidos no protocolo; e finalmente, (iii) a etapa de extração de dados, que permitiu examinar minuciosamente os estudos selecionados, a fim de entender a frequência da resistência ao etambutol em casos diagnosticados com tuberculose.

Os critérios utilizados para inclusão de um artigo nesta revisão foram: (a) trabalhos publicados e disponíveis integralmente em bases de dados científicas ou em versões impressas, (b) trabalhos recentes (publicados a partir de 2014) que já possuam aprovação pela comunidade científica, (c) trabalhos que abordem a frequência ou detecção de resistência ao etambutol em casos ou amostras por Mycobacterium tuberculosis, mesmo que este não seja o tema principal do trabalho.

Foram excluídos aqueles que apresentassem avaliações sem o método utilizado, que não estudaram o etambutol, que não apresentam dados consistentes sobre este medicamento em relação a resistência ou detecção da resistência em casos ou amostras por Mycobacterium tuberculosis.

\section{RESULTADOS E DISCUSSÃO}

A Quadro 1 tem por propósito mostrar uma síntese dos artigos analisados, com base em uma revisão bibliográfica, a qual abrange estudos de diversos países, os quais estão localizados nos continentes Americano, Africano, Asiático e Europeu.

A análise incluiu os resultados apresentados quanto a resistência ao etambutol. Cada um dos artigos foi analisado individualmente e, posteriormente, foi apresentada uma análise global de todos os trabalhos, levando em consideração os tópicos relevantes sobre a frequência da resistência ao etambutol. 
Quadro 1 - Resumo dos artigos analisados.

\begin{tabular}{|c|c|c|}
\hline Autor(es) & País & Principais Resultados \\
\hline Al-Mutari NM, et al. (2019) & Kuwait & $\begin{array}{l}\text { De } 242 \text { casos, } 64 \text { apresentam resistência a todos } \\
\text { os quatro medicamentos (Rifampicina, Isoniazida, } \\
\text { Estreptomicina e Etambutol), } 26 \text { à Isoniazida e } \\
\text { Etambutol; } 11 \text { à Isoniazida, Estreptomicina e } \\
\text { Etambutol }\end{array}$ \\
\hline Coban AY, et al. (2019) & Turquia & $\begin{array}{l}\text { De } 140 \text { Casos, } 23 \text { apresentam resistência à } \\
\text { Etambutol e Estreptomicina; } 4 \text { à Etambutol }\end{array}$ \\
\hline Cui Z, et al. (2014) & China & $\begin{array}{llll}\text { De } 767 & \text { Casos, } 275 & (35,8 \%) & \text { apresentam } \\
\text { resistência à Etambutol } & & \\
\end{array}$ \\
\hline Feliciano CS, et al. (2018) & Brasil e Moçambique & $\begin{array}{l}\text { A sensibilidade e especificidade do WGS para } \\
\text { detectar mutações associadas com resistência } \\
\text { foram respectivamente: } 100 \% \text { e } 77,2 \% \text { para } \\
\text { etambutol. }\end{array}$ \\
\hline Giri A, et al. (2018) & Índia & $\begin{array}{l}\text { De } 360 \text { casos, } 29 \text { (8\%) apresentam resistência à } \\
\text { Etambutol }\end{array}$ \\
\hline Gupta A, et al. (2015) & Índia & $\begin{array}{l}\text { De } 354 \text { casos, } 113 \text { apresentam resistência à } \\
\text { Etambutol, sendo } 16 \text { mono resistente, } 86 \text { à } \\
\text { Etambutol e Isoniazida e } 76 \text { à Etambutol e } \\
\text { Rifampicina }\end{array}$ \\
\hline Kerubo G, et al. (2016) & Quênia & $\begin{array}{l}\text { De } 184 \text { Casos, 25(13,5\%) apresentam resistência } \\
\text { à Etambutol }\end{array}$ \\
\hline Kim HJ, et al. (2017) & Coreia & $\begin{array}{l}\text { De } 2393 \text { Casos, } 88 \text { (3,7\%) apresentam resistência } \\
\text { à Etambutol. }\end{array}$ \\
\hline Li Y, et al. (2016) & China & $\begin{array}{l}\text { De } 1017 \text { Casos, } 63 \quad(6,19 \%) \text { apresentam } \\
\text { resistência à Etambutol, sendo } 3(0,2 \%) \text { somente } \\
\text { à Etambutol }\end{array}$ \\
\hline Li Q, et al. (2018) & China & De 1020 casos, $15(1,5 \%)$ à Etambutol. \\
\hline Namburete El, et al. (2016) & Moçambique & $\begin{array}{l}\text { De } 125 \text { Casos, } 8 \text { apresentam resistência à } \\
\text { somente Etambutol e } 1 \text { à Fluoroquinolona e } \\
\text { Estreptomicina }\end{array}$ \\
\hline Mao X et al. (2015) & China & $\begin{array}{l}\text { MTBDRsI não é uma escolha apropriada para } \\
\text { detecção de resistência ao etambutol }\end{array}$ \\
\hline Xu Y et al. (2015) & China & $\begin{array}{l}\text { De } 1048 \text { Casos, } 109 \text { apresentam resistência à } \\
\text { Etambutol }\end{array}$ \\
\hline Zeng $X$ et al. (2017) & China & $\begin{array}{l}\text { De } 162 \text { Casos, }(1,2 \%) \text { apresentam resistência à } \\
\text { Etambutol }\end{array}$ \\
\hline
\end{tabular}

Fonte: Alcântara RT, et al., 2019.

Feliciano CS, et al. (2018) em seu estudo transversal avaliou a resistência do M. tuberculosis ao tratamento com pacientes do sudeste do Brasil e da região central de Moçambique, observando às mutações mais frequentes. Foi possível identificar após a análise que o tipo de mutação relacionada à resistência ao etambutol foi diferente entre os países. Sendo o embB Met306Val e o embB Met306lle mais frequente entre os pacientes brasileiros e o embB Gln497Arg entre os moçambicanos. Além disto, foi encontrado na totalidade de casos do estudo sensibilidade e especificidade do WGS para detectar mutações associadas com resistência para o etambutol $100 \%$ e $77,2 \%$, respectivamente. 
De acordo com Cui Z, et al. (2014), ao analisar a forma de diagnóstico para a tuberculose, constata-se que ainda são poucos os testes moleculares para determinar a resistência ao Etambutol e as outras drogas utilizadas no tratamento da tuberculose. Logo, apesar de ter encontrado em seu estudo realizado na China mutações ao embC-embA, podem existir diferentes mutações ainda não identificadas em outros países. Sendo que dos casos analisados em sua pesquisa $35,8 \%$ apresentou a mutação ao embC-embA e consequentemente resistência ao tratamento.

O estudo realizado por Kerubo et al. (2016), no Quênia coletou 223 amostras de escarro de pacientes com baciloscopia positiva para tuberculose pulmonar (PTB), 184 amostras foram válidas para o estudo, dos quais, $87 \%$ dos pacientes estavam sendo tratados pela primeira vez para a tuberculose e $13 \%$ já tinham passado por tratamento anteriormente. Do total de casos, 25 apresentaram resistência ao Etambutol. Sendo o medicamento com maior prevalência de resistência a Isoniazida. Ainda se observou uma resistência a múltiplas drogas em $4,4 \%$ dos casos que estavam sendo tratados pela primeira vez. No entanto, não se obteve nenhuma diferença significativa quanto a proporção de resistência por sexo e idade.

Gupta A, et al. (2015) também observou a implicação das mutações genéticas na resistência ao etambutol (EMB). O autor estudou a ocorrência da mutação do embB 306 em cepas que apresentavam a resistência e sensibilidade ao EMB. Dos 354 casos analisados no estudo realizado na Índia mais de 30\% apresentou resistência ao EMB por mutação do embB 306. Ao analisar os dados obtidos na pesquisa pode-se concluir que o embB 306 além de ser a principal mutação causadora da resistência ao EMB, também pode ser um marcador sensível à resistência.

Em estudo realizado com crianças e adolescentes coreanos por Kim HJ, et al. (2017), de 2007 a 2013. Dos 2.690 pacientes, dos quais 297 foram excluídos devido a dados insuficientes, resultando em 2.393 casos, analisou-se a susceptibilidade da Mycobacterium tuberculosis ao tratamento da tuberculose. Obtendo-se, desta forma, 13,5\% dos apresentando resistência a um ou mais medicamentos usados. Quando analisados de forma individual obteve-se os seguintes índices: isoniazida (INH) $10,2 \%$, rifampicina (RFP) $5,1 \%$, etambutol (EMB) 3,7\% e pirazinamida (PZA) 3,1\%. Sendo o etambutol o terceiro medicamento com maior resistência.

Li Y, et al. (2016) realizou o estudo em diversas cidades da China, tendo como resultado em comum que a multirresistência, ou seja, resistência ao etambutol e aos outros medicamentos utilizados no tratamento da tuberculose, é maior em pacientes que estão sendo submetidos novamente ao tratamento. Da análise geral de seu trabalho, dos 1020 casos da amostra, 15(1.5\%) tinham resistência isolada ao etambutol. Ao realizar um comparativo, no ano de 2011 de 1184 casos, 60 (5.1\%) tiveram resistência isolada ao etambutol, destes 13 foram casos novos e 47 casos já tratados e, no ano de 2015 dos 1326 da amostra, 38 (2.9\%) tiveram resistência isolada ao etambutol, destes 15 foram casos novos e 23 casos já tratados. O presente estudo mostra que nos últimos 5 anos existem pesquisas que procuram esclarecer a respeito da resistência ao etambutol por Mycobacterium tuberculosis.

Em estudos realizados por Li Q et al. (2018); Namburete El et al. (2016), foi constatado resistência ao etambutol em pacientes que já haviam sido tratados para tuberculose. Para Cui Z et al. (2014); Namburete El et al. (2016), a resistência ao fármaco em questão está relacionada a presença de determinadas mutações genéticas encontradas através de testes moleculares.

Assim, segundo Feliciano CS et al. (2018); Namburete El et al. (2016); Zeng X et al. (2017) o teste molecular indicado é capaz de determinar tais mutações responsáveis pela resistência ao etambutol é o MTBDRsl, porém, mesmo o gene mais prevalente na mono resistência (embB) só esteve presente em $90 \%$ destes casos, conforme Namburete El et al. (2016), o que nos faz pensar em outros genes que desencadeiam tal resistência. No entanto, X. M et al. (2015) em sua meta-análise, relata que o MTBDRsI não é a escolha mais apropriada para detecção de resistência a etambutol.

Desta forma, novos testes estão sendo utilizados para se conseguir detectar tal resistência. A Descoloração Violeta de Cristal é outro teste que apresenta boa sensibilidade e especificidade na pesquisa de resistência ao etambutol. (COBAN AY et al., 2019). Outro teste é o WGS, que possuindo uma sensibilidade 
de $100 \%$, porém uma especificidade de $77,2 \%$ para o etambutol. (FELICIANO CS et al., 2018). Desta forma, em todos os resultados encontrados, o etambutol se encaixaria como o fármaco com baixa frequência de resistência. No entanto, mesmo em países com uma baixa incidência de tuberculose (24 casos por 100.000), como o Kuwait foi possível observar uma resistência ao etambutol (AL-MUTAIRI NM et al., 2019).

\section{CONSIDERAÇÕES FINAIS}

Os estudos revisados permitiram uma visão abrangente sobre a frequência da resistência ao etambutol em pacientes com tuberculose ou amostras de Mycobacterium tuberculosis. Como pode ser visto, esta é uma pesquisa atual e grande parte dos estudos foram publicados nos últimos cinco anos. As análises foram realizadas em literaturas de diferentes países e continentes, tendo dentre eles o Brasil. Em suma, foi possível concluir que a frequência em que encontramos resistência ao etambutol em casos com tuberculose ainda é baixa, quando comparado aos demais medicamentos que fazem parte do esquema de primeira escolha para o tratamento da doença. Dentre os trabalhos analisados o com maior frequência para resistência apresentou em volta de $40 \%$. Porém, devido às limitações atuais de testes para detecção de resistência ao etambutol, devemos considerar que os resultados aqui apresentados podem ser maiores do que os observados e que 0 etambutol sofra uma resistência ainda maior.

\section{REFERÊNCIAS}

1. AL-MUTAIRI N, et al. Occurrence of disputed rpoB mutations among Mycobacterium tuberculosis isolates phenotypically susceptible to rifampicin in a country with a low incidence of multidrug-resistant tuberculosis. BMC Infectious Diseases, 2019; 19: 1-3.

2. COBAN A, et al. Nitrate reductase assay for rapid detection of isoniazid, rifampin, ethambutol, and streptomycin resistance in Mycobacterium tuberculosis: a systematic review and meta-analysis. Journal of clinical microbiology, 2014; 52: 15-9.

3. COBAN A, et al. A new colorimetric method for rapid detection of ethambutol and streptomycin resistance in Mycobacterium tuberculosis: crystal violet decolorization assay (CVDA). Antonie van Leeuwenhoek, 2019; 112: 679685.

4. CUI Z, et al. Mutations in the embC-embA intergenic region contribute to mycobacterium tuberculosis resistance to ethambutol. Antimicrobial Agents and Chemotherapy, 2014; 58: 6837-6843.

5. FELICIANO C, et al. Accuracy of whole genome sequencing versus phenotypic (MGIT) and commercial molecular tests for detection of drug-resistant Mycobacterium tuberculosis isolated from patients in Brazil and Mozambique. Tuberculosis (Edinburgh, Scotland), 2018; 110: 59-67.

6. FENG Y, et al. Rapid diagnosis of drug resistance to fluoroquinolones, amikacin, capreomycin, kanamycin and ethambutol using genotype MTBDRsl assay: a meta-analysis. PloS one, 2013; 8: e55292.

7. GIRI A, et al. Polymorphisms in Rv3806c (ubiA) and the upstream region of embA in relation to ethambutol resistance in clinical isolates of Mycobacterium tuberculosis from North India. Tuberculosis (Edinburgh, Scotland), 2018; 108: 41-46.

8. GUPTA A, et al. Mutations at embB306 codon and their association with multidrug resistant M. tuberculosis clinical isolates. Indian journal of medical microbiology, 2015; 33: 387-92.

9. KERUBO G, et al. Drug susceptibility profiles of pulmonary Mycobacterium tuberculosis isolates from patients in informal urban settlements in Nairobi, Kenya. BMC infectious diseases, 2016; 16: 583.

10. $\mathrm{KIM} \mathrm{H}$, et al. The Rate of Drug-Resistant Tuberculosis in Korean Children and Adolescents Since 2007. Journal of Korean medical science, 2017; 32: 954-960.

11. LI Q, et al. Prevalence and patterns of drug resistance among pulmonary tuberculosis patients in Hangzhou, China. Antimicrobial resistance and infection control, 2018; 7:61.

12. LI Y, et al. Association between embB Codon 306 Mutations, Phenotypic Resistance Profiles, and Genotypic Characterization in Clinical Mycobacterium tuberculosis Isolates from Hebei, China. Antimicrobial agents and chemotherapy, 2016; 60: 7295-7302.

13. KASPER I, et al. Medicina Interna de Harrison. 19th ed. Porto Alegre: AMGH, 2017; 2770 p.

14. NAMBURETE E, et al. Drug-resistant tuberculosis in Central Mozambique: The role of a rapid genotypic susceptibility testing. BMC Infectious Diseases, 2016; 16: 17. 
15. NOGUEIRA AF, et al. Tuberculosis: a general approach of the main aspects. Brazilian Journal of Pharmacy, 2012; $3: 8$.

16. REZAEI $F$ et al. Frequency of mutational changes in the embB among the ethambutol-resistant strains of Mycobacterium tuberculosis in Iran. Journal of infection in developing countries, 2016; 10: 363-8.

17. SAFI $\mathrm{H}$ et al. Evolution of high-level ethambutol-resistant tuberculosis through interacting mutations in decaprenylphosphoryl- $\beta$-D-arabinose biosynthetic and utilization pathway genes. Nature genetics, $2013 ; 45: 1190-7$.

18. SANGARÉ $L$ et al. Mycobacterium tuberculosis drug-resistance in previously treated patients in Ouagadougou, Burkina Faso. Annals of African Medicine, 2010; 9: 15-19.

19. SANGARÉ $L$ et al. Anti-tuberculosis drug resistance in new and previously treated pulmonary tuberculosis cases in Burkina Faso. The international journal of tuberculosis and lung disease: the official journal of the International Union against Tuberculosis and Lung Disease, 2010; 14: 1424-9.

20. SILVA MEND, et al. General aspects of tuberculosis: an update on the etiologic agent and treatment.Brazilian Journal of Clinical Analyses, 2018; [s.n].

21. TAKAYAMA K et al. Inhibition by ethambutol of mycolic acid transfer into the cell wall of Mycobacterium smegmatis. Antimicrobial Agents and Chemotherapy, 1979; 16: 240-242.

22. WORLD HEALTH ORGANIZATION. Global tuberculosis report 2013. WHO Library Cataloguing-in-Publication Data. 2013.

23. WORLD HEALTH ORGANIZATION. Global Tuberculosis ReportBlood. [s.l: s.n.].

24. WU MH et al. Proficiency of drug susceptibility testing for Mycobacterium tuberculosis in Taiwan, 2007-2011. International Journal of Tuberculosis and Lung Disease, 2014; 17: 1.

25. MAO X, et al. Diagnosis of drug resistance to fluoroquinolones, amikacin, capreomycin, kanamycin and ethambutol with genotype MTBDRsl assay: A meta-analysis. Annals of Clinical and Laboratory Science, 2015; 45: 533-544.

26. $\mathrm{XU} \mathrm{Y}$, et al. Mutations Found in embCAB, embR, and ubiA Genes of Ethambutol-Sensitive and -Resistant Mycobacterium tuberculosis Clinical Isolates from China. BioMed Research International, 2015; 2015: 8.

27. ZENG X, et al. Performance of the MTBDRsI Line probe assay for rapid detection of resistance to second-line antituberculosis drugs and ethambutol in China. Diagnostic Microbiology and Infectious Disease, 2017; 89: $112-117$. 